\title{
Life and Death in a Gang-A Mathematical Model of Gang Membership
}

\author{
J. Sooknanan ${ }^{1}$, B. Bhatt ${ }^{1} \&$ D. M. G. Comissiong ${ }^{1}$ \\ ${ }^{1}$ Department of Mathematics and Statistics, The University of the West Indies, St. Augustine, Trinidad and Tobago \\ Correspondence: D. M. G. Comissiong, Department of Mathematics and Statistics, The University of the West In- \\ dies, St. Augustine, Trinidad and Tobago. Tel: 1868-662-2002 Ext. 83099. E-mail: Donna.Comissiong@sta.uwi.edu
}

Received: June 1, 2012 Accepted: June 17, 2012 Online Published: July 4, 2012

doi:10.5539/jmr.v4n4p10 URL: http://dx.doi.org/10.5539/jmr.v4n4p10

\begin{abstract}
There is increasing gang membership worldwide, but few mathematical models are available describing the growth of gangs in a population. A mathematical model consisting of a system of four coupled, nonlinear ordinary differential equations is used to divide a population into four groups based on gang affiliation. Demographics and vertical transmission are included in the model. One of the main findings of this research is the conditions under which the gang members become extinct. Bifurcation analyses shows the existence of "tipping points" in the murder rates, gang recruitment rates and the imprisonment and deterrence rates.
\end{abstract}

Keywords: gangs, nonlinear dynamical systems, peer contagion, equilibria

\section{Introduction}

Gangs are involved in a wide variety of crimes and are fast becoming an increasingly worldwide occurrence (Hagedorn, 2005). Trinidad and Tobago is no stranger to the gang phenomenon, especially since its proximity to South America makes it a prime transshipment point for drugs and guns. There is a well established local link between violent crime, gangs, guns and the illegal drug trade (Katz, 2009) (Trinidad and Tobago House of Representatives, Parlimentary session January 2008). The development of strategies and public policies to stem the rising crime rate is vital and therein lies the strength of mathematical modelling. Mathematical modelling and numerical simulation augment the traditional approaches to research since they help organise existing data, identify areas with missing data and are relatively inexpensive and more practical than carrying out an actual experiment (Castiglione, 2006). However, policy makers are susceptible to what Ball (2003) calls linear thinking and this has led to the development of linear models of human behavior. Yet human behaviour is inherently nonlinear (Brown, 1995), and thus we assume that criminal behaviour and crime may best be described by a nonlinear system.

The choice of an infectious disease model is motivated by research suggesting that "the best model for violence may be that of a socially infectious disease" (Bingenheimer, 2005). Gang membership is treated as an infection that multiplies due to interaction or peer contagion whereby "delinquent youth co-opt at-risk youth through verbal and non-verbal communication" (Katz, 2010). Accordingly, a deterministic, infectious disease model which divides the population into compartments based on their allegiance to the gang is used to analyse how the number of gang members may be affected by various crime-fighting policy changes. In addition, the deterministic approach is useful in detecting bifurcation points (tipping points) of the system where the stability is suddenly lost, and the system makes a quick transition to a new, very different behavior.

The model uses as a starting point a model describing violent crime and burglary (Ormerod et al., 2001; Campbell \& Omerod, 1997). The model is, to the authors' knowledge, the only application of an infectious disease model to crime. However, the model does not explicitly consider the subset of gangs with its own characteristic dynamics.

The paper is organized as follows. We begin by providing a description of the model. The different equilibria and their stability are then analysed. After estimating the parameters utilized in the model, these values are used to determine the end states of the system, where the parameters are changed to reflect possible anti-gang strategies. After a brief discussion, we conclude by suggesting possible extensions to our model. 


\section{The Model}

A system of four differential equations is used to divide a population $(T)$ into four disjoint groups or classes $(N, S, G$, and $R$ ) who differ in their allegiances to the gang lifestyle. $N$ represents those who are currently not susceptible to a life in the gang, but are potential gang members. The gang members are represented by the $S$ and $G$ groups where $S$ represents the fringe membership and $G$ represents the core membership. Core members consist generally of the older gang members who have made a personal commitment to the gang culture (Klein, 1995). Fringe members are the entry level members who are less involved in gang crime and are not yet fully committed to the gang lifestyle (Klein, 1995). $R$ represents the gang members who are in jail. The model is analogous to models used in mathematical epidemiology to describe the spread of an infection in a population. The strength of the flows between the groups over time are related to factors identified in the literature related to the formation and desistance from gangs such as social and economic conditions, intervention strategies and the deterrent effects of the criminal justice system.

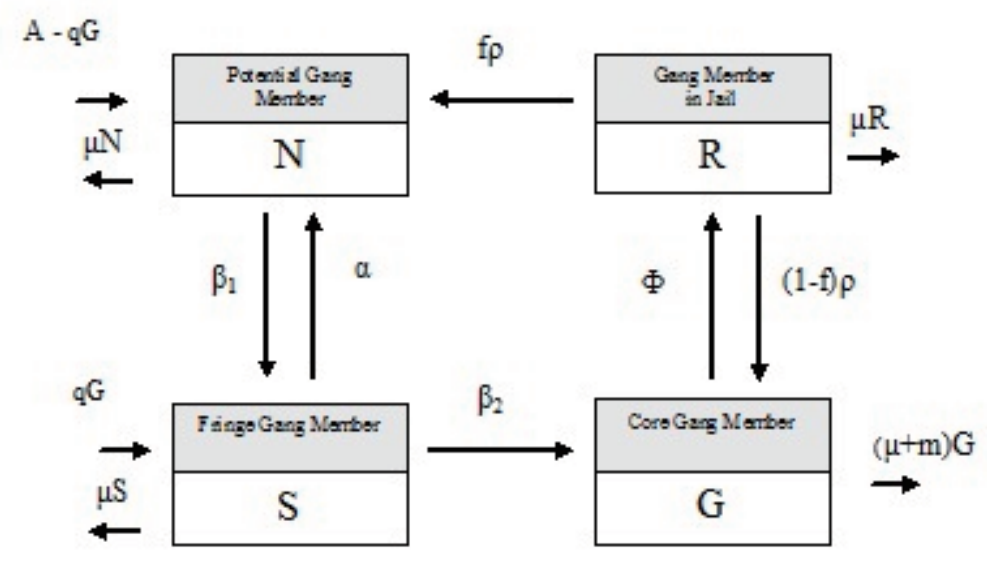

Figure 1. Representation of model

Figure 1 shows the structure of flows and parameters used within the model which is described by following non-linear system of equations:

$$
\begin{gathered}
\frac{d N}{d t}=A-q G-\beta_{1}(S+G) \frac{N}{T}+\alpha S+f \rho R-\mu N \\
\frac{d S}{d t}=q G+\beta_{1}(S+G) \frac{N}{T}-\beta_{2} \frac{S G}{T}-(\alpha+\mu) S \\
\frac{d G}{d t}=\beta_{2} \frac{S G}{T}+(1-f) \rho R-(\Phi+m+\mu) G \\
\frac{d R}{d t}=\Phi G-(\rho+\mu) R \\
\frac{d T}{d t}=A-(\mu T+m G) \\
T=N+S+G+R
\end{gathered}
$$

where: $\beta_{1}$ is the contact rate which results into a conversion to a fringe gang member; $\beta_{2}$ is the conversion rate to core gang members; $\alpha$ is the intervention/deterrence parameter for fringe members; $\Phi$ is the imprisonment rate; $1-f$ is the recidivism/ relapse rate; $\rho$ is the (average length of jail sentence) $)^{-1} ; q$ is the proportion of population who are "born" fringe members; $m$ is the murder rate of gang members; $A$ is the number of incoming youth into the population; and $\mu$ is the natural death rate.

The model incorporates births $A$, deaths-natural $\mu$ and murders $m$-and vertical transmission. Vertical transmission in epidemiology is another mode of disease transfer where a disease is transmitted to an unborn or newly born 
child from its parent. Using this analogy with gangs, some youth appear predestined for gang membership. This may be as a result of family or friends who are involved in criminal activity or neighborhood traditions where gang membership and violence are regarded as a normal part of growing up (Nawojczyk, 1997; Moore, 1978). To incorporate vertical transmission in the model, it is assumed that a fraction $q$ of the relatives or friends from the core gang group are born into the fringe group. Thus $A-q G$ members are born into the potential gang members class.

Potential members $(N)$ may by contact $\left(\beta_{1}(S+G) \frac{N}{T}\right)$ with gang members become a fringe member $(S)$. Since fringe members are not yet committed to the gang, they may be swayed from this lifestyle-thus the model includes an intervention/ interference parameter $\alpha$ at this stage. Despite these intervention measures, contact between the fringe and core members $\left(\beta_{2} \frac{S G}{T}\right)$ may result in additional core gang members $(G)$. We assume that $\beta_{1} \geqslant \beta_{2}$, so that it is easier to convince someone to become a fringe member $\left(\beta_{1}\right)$ than it is to persuade someone who is a fringe member to take the final step and commit to the gang $\left(\beta_{2}\right)$. These gang members $(G)$ may then go to jail with an imprisonment rate of $\Phi$. On completion of their their jail sentence, there are two possibilities. Jailed members may have been rehabilitated and recover to join the potential $(N)$ class or there may be a relapse to the $G$ group. $\rho^{-1}$ gives the average length of the jail sentence. The proportion of prisoners moving back to $G$ after release is defined as $(1-f)$, hence $(1-f) \rho$ denotes the recidivism or relapse rate while $f \rho$ denotes the rehabilitation rate.

People in the $G$ category cannot leave the gang voluntarily since they have knowledge of the inner workings of the gang and are most likely identified as gang members. Hence protection from retaliation by rival gangs and stigmatisation may be factors in the decision to stay in a gang (Hastings et al., 2011). There are three key motivating factors to leaving the gang-maturity, aging and violence (both committed by the gang member and directed towards him and his family)(Hastings et al., 2011). While we acknowledge this research, this model does not consider a voluntary flow out of the gang. Thus the only way to exit the $G$ category is to go to jail with an imprisonment rate of $\Phi$ or to die. All these parameters are considered as non-negative since negative solutions have no epidemiological significance. In keeping with the infectious disease analogy, we consider there to be no incubation period for the infection of gang membership. Due to the perceived status of the gang lifestyle, we consider an individual who is in the gang as immediately infectious and able to infect others to join the gang.

We assume that

$$
N(0) \geqslant 0, S(0) \geqslant 0, G(0) \geqslant 0, R(0) \geqslant 0 .
$$

The parameter values were calculated from available data (details of these calculations are given in the Appendices), and initial conditions were chosen from the literature on gangs to reflect the local situation. The behaviour of the model is investigated through stability analysis and numerical simulation. In order to explore possible bifurcation points we use continuation methods in the graphical Matlab package MATCONT.

\section{Analysis}

\subsection{Calculation of $R_{0}$}

The issue of whether or not a disease can invade a host population and persist or remain endemic involves the introduction of a threshold-the basic reproductive number $R_{\mathbf{0}}$-the average number of secondary cases caused by an infected individual. $R_{\mathbf{0}}$ is calculated using the next generation operator method (Castillo-Chavez et al., 2002). In applying this method, $S$ and $G$ are considered the infective compartments, $N$ represents the non-infective compartments and $R$ represents individuals who are infected but do not transmit the disease.

The Jacobian at $(N, S, G, R)=\left(\frac{A}{\mu}, 0,0,0\right)$ is

$$
\left[\begin{array}{cc}
\beta_{1}-(\alpha+\mu) & \beta_{1}+q \\
0 & \frac{(1-f) \rho \Phi}{\rho+\mu}-(\Phi+m+\mu)
\end{array}\right] .
$$

This is re-written in the form $M-D$ such that $m_{i j}>0$ and D with $d_{i j}>0$ is a diagonal matrix. We use

$$
M=\left[\begin{array}{cc}
\beta_{1} & \beta_{1}+q \\
0 & 0
\end{array}\right]
$$

and

$$
D=\left[\begin{array}{cc}
\alpha+\mu & 0 \\
0 & (\Phi+m+\mu)-\frac{(1-f) \rho \Phi}{\rho+\mu}
\end{array}\right] .
$$


We take $R_{0}$ to be the dominant eigenvalue of $M D^{-1}$. Hence

$$
R_{0}=\max \left(\frac{\beta_{1}}{\alpha+\mu}, \frac{\Phi \rho(1-f)}{(\mu+\rho)(m+\Phi+\mu)}\right) .
$$

where

$$
\frac{\Phi \rho(1-f)}{(\mu+\rho)(m+\Phi+\mu)}<1 .
$$

Since the importance of $R_{0}$ lies in whether it is less than or greater than one, we use $R_{0}=\frac{\beta_{1}}{\alpha+\mu}$. The condition $R_{0}<1$ is, at least, a necessary condition for a globally asymptotically stable gang-free state. On the other hand, $R_{0}>1$ allows for the possibility of multiple stable coexistence states. In this model, the transmission may be considered a collective phenomenon-societal and peer pressure play important roles-so $R_{0}$ may be considered as indicative of the suitability of the environment to harbouring gangs-the ratio of how quickly people become the lower rank gang members to how quickly they can be dissuaded from that lifestyle.

\subsection{Disease Dynamics: Equilibria}

The equilibrium values (steady state of the system) provide insight into the long-term behavior or asymptotic properties of the system and can be used to determine if the system has periodic behavior or not i.e. if there are oscillations. These steady state solutions or end states are determined by setting the system of ODEs equal to zero. The stability properties of the equilibrium points can be determined by making a linear approximation to the actual equations and then examining the stability of the linear approximation. From equation $(4), R^{*}=\frac{\Phi G^{*}}{\rho+\mu}$. Substituting this in equation (3), we get either

$$
G^{*}=0
$$

or

$$
\frac{S^{*}}{T^{*}}=\frac{(\Phi+m+\mu)-(1-f) \frac{\Phi \rho}{\rho+\mu}}{\beta_{2}}
$$

where

$$
(\Phi+m+\mu)-(1-f) \frac{\Phi \rho}{\rho+\mu}>0 .
$$

We consider these cases in the following subsections.

\subsubsection{Case 1: Gang-free and Core-free Equilibrium}

In this case, $G^{*}=0$. From equation (4) and equation (5), $R^{*}=0$ and $T^{*}=\frac{A}{\mu}$. Substituting these in equation (2), we get

$$
S^{*}\left[\beta_{1} \frac{N^{*}}{T^{*}} \cdot-(\alpha+\mu)\right]=0 .
$$

Hence, we consider two cases: $S^{*}=0$ (referred to as Case 1a) or $\beta_{1} \frac{N^{*}}{T^{*}} .-(\alpha+\mu)=0$ (referred to as Case $\left.1 \mathrm{~b}\right)$.

\subsubsection{Gang-free Equilibrium}

When $S^{*}=0, G^{*}=0$, and $R^{*}=0$, then $N^{*}=\frac{A}{\mu}$. This is the Gang-free equilibrium in which there are no gang members and only potential gang members.

\subsubsection{Core-free Equilibrium}

Using equation (1) results in

$$
S^{*}=\frac{A}{\mu}\left(1-\frac{\alpha+\mu}{\beta_{1}}\right), G^{*}=0, R^{*}=0, N^{*}=\frac{\alpha+\mu}{\beta_{1}} \frac{A}{\mu},
$$

where $\beta_{1}>\mu+\alpha$, so $R_{0}>1$. This is the Core-free equilibrium in which there are no core gang members or gang members in jail. 


\subsubsection{Case 2: Co-existence Equilibrium}

In this case, we consider

$$
\frac{S^{*}}{T^{*}}=\frac{(\phi+\mu+m)-(1-f) X \rho}{\beta_{2}},
$$

where $X=\frac{\Phi}{\rho+\mu}>0$. For ease of calculation let

$$
K=\frac{S^{*}}{T^{*}}=\frac{(\phi+\mu+m)-(1-f) X \rho}{\beta_{2}} .
$$

Using equations (5) and (4), we get

$$
G^{*}=\frac{A-\mu T^{*}}{m} \text { and } R^{*}=X G^{*}=X\left[\frac{A}{m}-T^{*} \frac{\mu}{m}\right] .
$$

From equation (6), we obtain

$$
N^{*}=T^{*}(1-K+Z \mu)-Z A,
$$

where

$$
Z=\frac{1+X}{m}>0 \text { and } 1-K+Z \mu>0 .
$$

Substituting for $N^{*}, S^{*}, R^{*}, G^{*}$ in equation (2) leads to

$$
a T^{* 2}+b T^{*}+c=0
$$

where

$$
\begin{gathered}
a=K\left(\beta_{1}-\mu-\alpha\right)+K Z \mu \beta_{1}+\frac{K}{m} \mu \beta_{2}+\frac{K}{m} \mu \beta_{1}-\frac{1}{m} \mu \beta_{1}-\frac{Z}{m} \mu^{2} \beta_{1}-\frac{1}{m} q \mu-K^{2} \beta_{1}, \\
b=\frac{q A}{m}-\beta_{1} K Z A+\beta_{1} \frac{A}{m}(1-K+2 Z \mu)-\beta_{2} K \frac{A}{m}, \\
c=-\frac{\beta_{1} Z A^{2}}{m}<0 .
\end{gathered}
$$

For a solution to exist, $b^{2}-4 a c \geqslant 0$. There may be zero, one or two solutions depending on the values of $a, b$ and c. Hence

$$
S^{*}=K T^{*}, G^{*}=\frac{A-\mu T^{*}}{m}, R^{*}=X G^{*}=X\left[\frac{A}{m}-T^{*} \frac{\mu}{m}\right] .
$$

\subsection{Stability of Equilibria}

There are two ways to determine the stability of the system at the equilibrium values. The eigenvalues of the Jacobian at the point may be determined or the Routh Hurwitz condition may be used. The Jacobian of the system is

$$
J=\left[\begin{array}{cccc}
\frac{-\beta_{1}(S+G)}{T}-\mu & -\frac{\beta_{1} N}{T}+\alpha & -\frac{\beta_{1} N}{T}-q & f \rho \\
\frac{\beta_{1}(S+G)}{T} & \frac{\beta_{1} N}{T}-\frac{\beta_{2} G}{T_{\beta_{2}}}-(\alpha+\mu) & q+\frac{\beta_{1} N}{T}-\frac{\beta_{2} S}{T} & 0 \\
0 & \frac{\frac{\beta_{2}}{T}}{T}-(\Phi+m+\mu) & (1-f) \rho \\
0 & 0 & \Phi & -(\rho+\mu)
\end{array}\right] .
$$

Let us consider first the case of Gang-free equilibrium $S^{*}=0, G^{*}=0$. The gang-free equilibrium values

$$
\left(T^{*}, N^{*}, S^{*}, G^{*}, R^{*}\right)=\left(\frac{A}{\mu}, \frac{A}{\mu}, 0,0,0\right)
$$

are substituted into $J$. The algebraic expressions in the Jacobian matrix are simple enough to allow for a straightforward determination of the eigenvalues $\lambda$. The eigenvalues are:

$$
\beta_{1}-\mu-\alpha,-\mu, \frac{1}{2}\left[-\mu-(m+\Phi+\rho) \pm \sqrt{(m+\Phi+\rho)^{2}-4 f \Phi \rho-4 m \rho}\right] .
$$

Since $f, \Phi, \rho, m>0$,

$$
\sqrt{(m+\Phi+\rho)^{2}-4 f \Phi \rho-4 m \rho}<(m+\Phi+\rho) .
$$


Hence

$$
\operatorname{Re}\left[\frac{1}{2}\left(-\mu-(m+\Phi+\rho) \pm \sqrt{(m+\Phi+\rho)^{2}-4 f \Phi \rho-4 m \rho}\right)\right] \leqslant 0 .
$$

Thus if $\beta_{1}<\mu+\alpha$, the Routh-Hurwitz criteria are satisfied, and the gang-free equilibria is stable. This condition on the stability of the gang free equilibrium is the same as the condition that the basic reproduction number $R_{0}$ must be less than one. This means that once potential recruits can be dissuaded from joining a gang faster than they can be recruited and die naturally, no gangs will arise in the population.

Next we look at the case of Core-free equilibrium $S^{*} \neq 0, G^{*}=0$. The core-free equilibrium values are

$$
G^{*}=0, R^{*}=0, S^{*}=T^{*}\left(1-\frac{\alpha+\mu}{\beta_{1}}\right), N^{*}=\frac{\alpha+\mu}{\beta_{1}} T^{*}, T^{*}=\frac{A}{\mu},
$$

where $R_{0}>1$. Let us take

$$
\chi=\frac{\beta_{1}-(\alpha+\mu)}{\beta_{1}}, \quad \varepsilon=\Phi+m+\mu
$$

where $0<\chi<1$. The eigenvalues of $J$ are $-\mu,-\beta_{1} \chi$, and those of $\left[\begin{array}{cc}\beta_{2} \chi-\varepsilon & (1-f) \rho \\ \Phi & -(\rho+\mu)\end{array}\right]$.

In order for the eigenvalues of a $2 \times 2$ matrix to have negative real part, its determinant $\Delta$ must be positive and its trace $\tau$ must be negative. Since $\tau<0$ if

$$
\chi \beta_{2}<\Phi+2 \mu+\rho+m
$$

and $\Delta>0$ if

$$
(\Phi+m+\mu)(\rho+\mu)-(1-f) \rho \Phi>\beta_{2} \chi(\rho+\mu),
$$

it follows that when these conditions are satisfied, there will only be fringe gang members in the population.

Next, we consider the coexistence equilibrium. Substituting equation (9) into $J$ and simplifying using

$$
\varepsilon=\Phi+m+\mu, M=\frac{N^{*}}{T^{*}}, H=\frac{G^{*}}{T^{*}},
$$

we obtain a characteristic polynomial of the form

$$
\lambda^{4}+a_{1} \lambda^{3}+a_{2} \lambda^{2}+a_{3} \lambda+a_{4}=0
$$

where the expressions for $a_{1}, a_{2}, a_{3}$ and $a_{4}$ are provided in Appendix A. The Routh-Hurwitz criteria predict stability iff $a_{1}, a_{2}, a_{3}, a_{4}>0$ and $\left(a_{1} a_{2}-a_{3}\right) a_{3}>a_{1}^{2} a_{4}$. Owing to the complicated nature of these expressions, this analysis will be done by using the parameter values we have calculated.

\section{Estimating the Initial Conditions and Parameters}

One of the great difficulties in modelling is estimating values for the model parameters in order to determine model predictions. It must be emphasized at the onset that estimating the parameters in this model was extremely difficult due to the difficulty in quantifying parameters as well as difficulties in obtaining police and sociological records due to the sensitive nature of the research. Since some of the parameters can only be roughly estimated, the model is used not "to predict actual data but to explain stylized facts and understand the consequences of different hypothesis" (Gordon, 2010), as well as to suggest further data-gathering. In addition to estimating the parameters, the initial conditions $N(0), S(0), G(0), R(0)$ must be estimated for use as initial values in the numerical ODE solvers. The values are shown in Table 1 and Table 2. A detailed explanation of the estimation of each value can be found in Appendix B. 
Table 1. The parameter values

\begin{tabular}{cc}
\hline Parameter & Value \\
\hline$\beta_{1}$ & 0.71 \\
$\beta_{2}$ & 0.21 \\
$\alpha$ & 0.1265 \\
$\Phi$ & 0.115 \\
$\rho$ & 0.2 \\
$f$ & 0.44 \\
$q$ & 0.0145 \\
$A$ & 750 \\
$m$ & 0.0503 \\
$\mu$ & $2.585 \times 10^{-3}$ \\
\hline
\end{tabular}

Table 2. The initial conditions

\begin{tabular}{cc}
\hline Class & Initial Value \\
\hline$T(0)$ & 20,000 \\
$N(0)$ & 7464 \\
$S(0)$ & 11092 \\
$G(0)$ & 800 \\
$R(0)$ & 644 \\
\hline
\end{tabular}

Case 1 was found to be unstable for these parameter values. This means that the system cannot tend to the state of having no gang members (i.e. $G^{*} \neq 0$ ). Instead, it attains a coexistence endemic equilibrium where

$$
T^{*}=55814, N^{*}=9213, S^{*}=27721, G^{*}=12043, R^{*}=6836 .
$$

The eigenvalues were found to be:

$$
-0.5633,-0.0275+0.0346 i,-0.0275-0.0346 i,-0.2407 \text {. }
$$

Since the eigenvalues are all negative, the system is stable. This means that the system tends to an increase in the number of gang members from 800 to $G^{*}=12043$. Also, $R_{0}=\frac{\beta_{1}}{\alpha+\mu}>1$.

\section{Results}

The graph in Figure 2 shows the endemic equilibrium obtained using these parameter values. The end state values approximate the values found above by calculation. With the current parameter values, there is an increase in all the subpopulations. Various parameters are changed and the effect of these changes are now examined to determine which change would result in the greatest decrease in gang members. When the length of prison sentences $(\rho)$ is increased by $100 \%$, there is an increase in fringe members of $30 \%$ and a decrease in core members of $7 \%$ Decreasing the recidivism rate by about $60 \%$, results in an increase in fringe members of $140 \%$, a decrease in core members of $20 \%$. However, when we vary the interference parameter ( $\alpha$ which is related to $\Phi$ ), an increase of $25 \%$ leads to a $10 \%$ drop in the number of core gang members and a decrease in fringe gang members of $36 \%$. An increase of $50 \%$ leads to an increase in fringe members of $200 \%$ and a decrease in core members of $40 \%$. An increase in the imprisonment rate (and hence deterrence) of $25 \%$ with the length of prison sentences $(\rho)$ doubled leads to a an increase in fringe members of $110 \%$ and a decrease in core members of $20 \%$.

We now turn our attention to Figures 3 through 8 . We observe that improving the criminal justice system by increasing the imprisonment rate (and hence deterrence) by $25 \%$, and doubling the length of prison sentences ( $\rho$ ) as well as a $25 \%$ decrease in recidivism rate leads to an increase in fringe members of $200 \%$ and a decrease in core members of $35 \%$ (Figure 3). Reducing the contact rate $\beta_{1}$ by $28 \%$ does not have much of an effect on gang members- a $4 \%$ decrease in the core and a $16 \%$ decrease in the fringe gang members (Figure 4 ). Decreasing $\beta_{2}$ by the same amount results in a slower increase in the number of gang members reducing the number of core gang members ultimately by $20 \%$ and increasing fringe members by $120 \%$ (Figure 5). If we combine these measures 
in turn with improving the criminal justice system, by reducing $\beta_{1}$ by $40 \%$, this leads to the no-core members equilibrium (Figure 6). Combining the improvement of the criminal justice system with reducing $\beta_{2}$ by $20 \%$ leads to the Core-free equilibrium (Figure 7). Changing all the parameters by $25 \%$ in combination and doubling the jail sentences leads to the gang-free equilibrium (Figure 8).

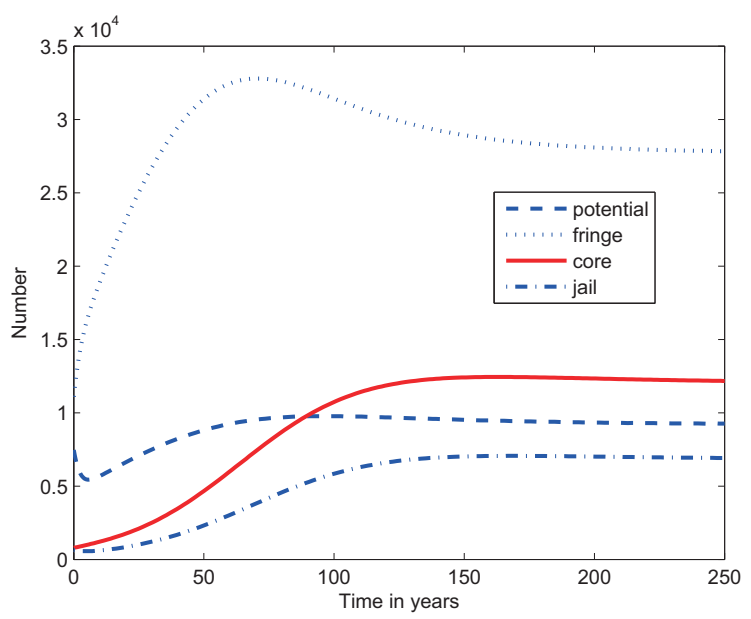

Figure 2. Coextinction of all groups for model parameters

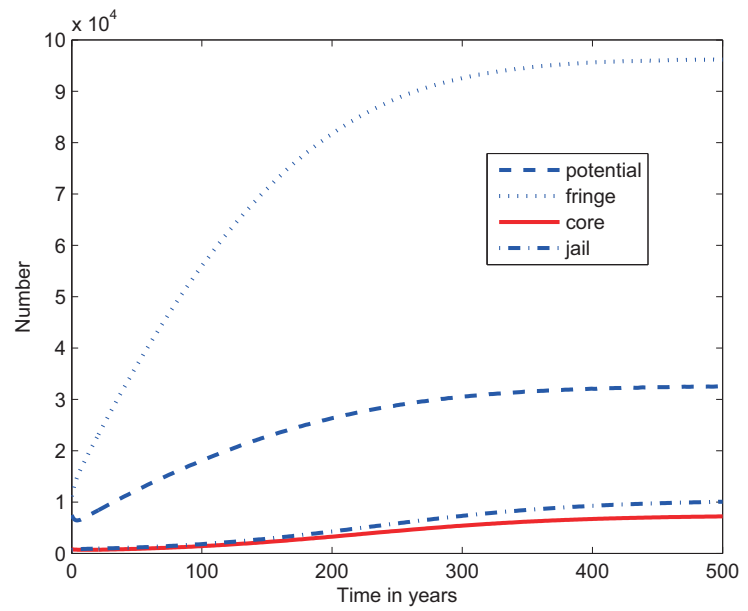

Figure 3. The criminal justice system

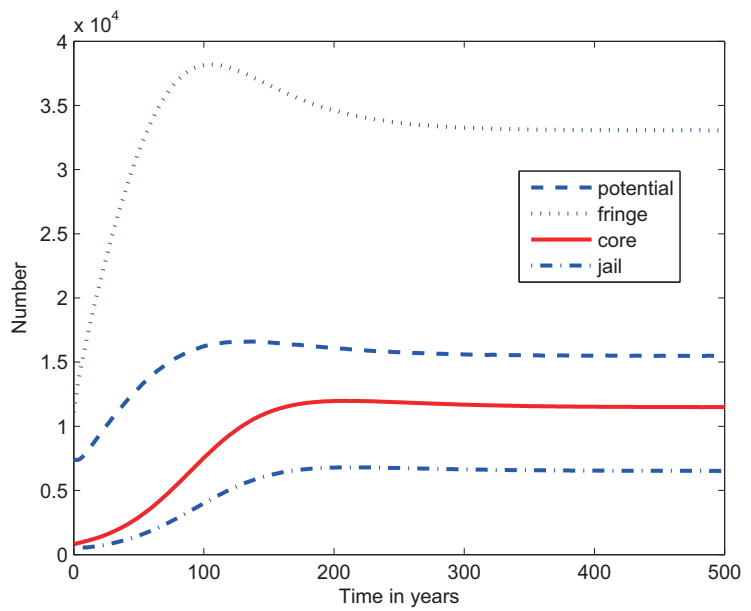

Figure 4. Reducing $\beta_{1}$ by $28 \%$ 


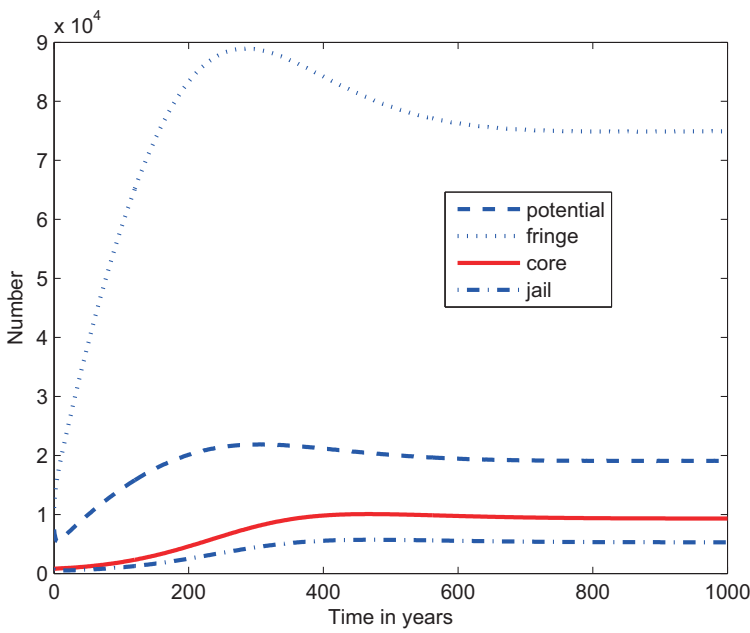

Figure 5. Changing all the parameters by $25 \%$

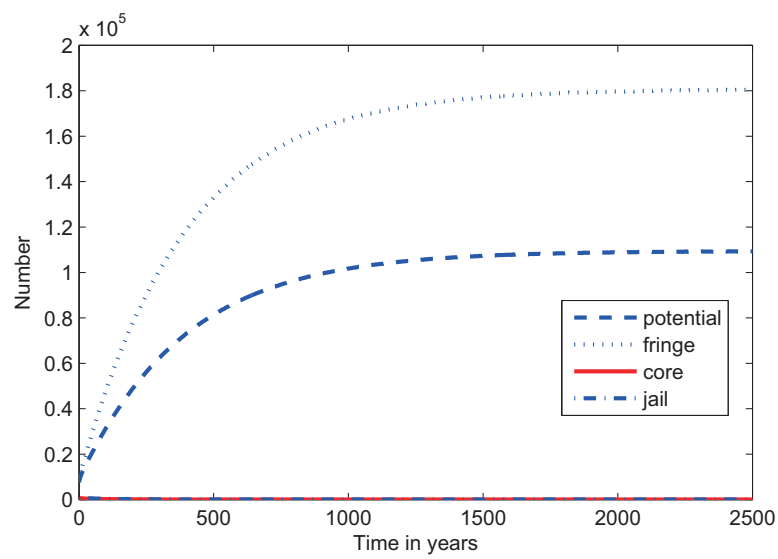

Figure 6. Reducing $\beta_{1}$ and improving the criminal justice system

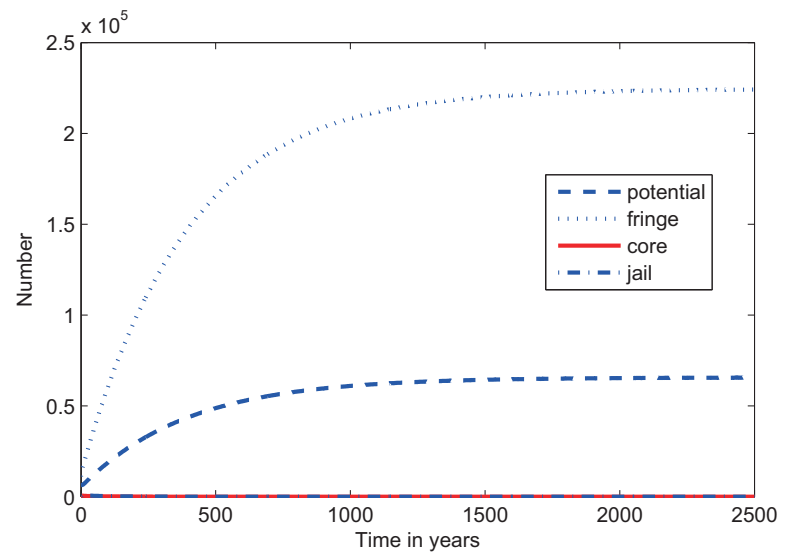

Figure 7. Reducing $\beta_{2}$ and improving the criminal justice system 


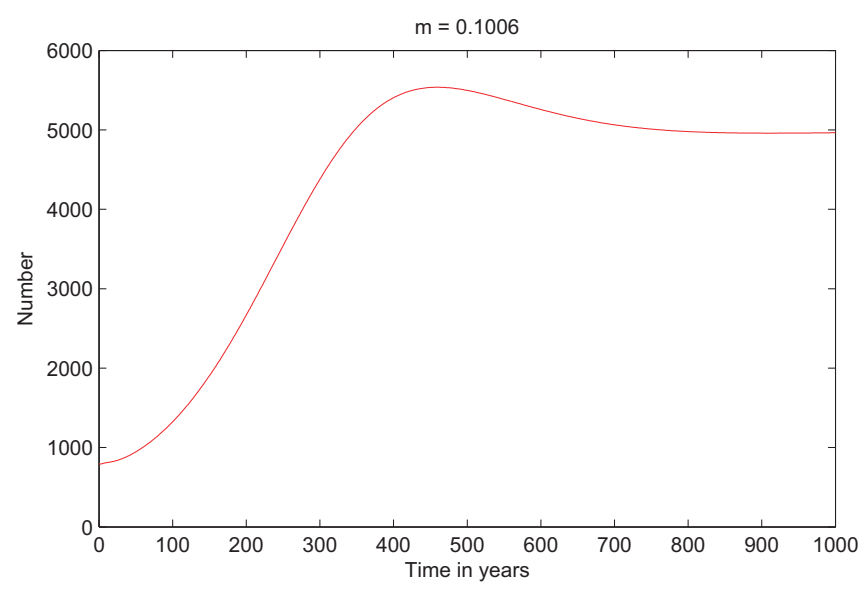

Figure 8. Graph showing the effect of $2 * m$ on $G$

\section{Bifurcation Analysis}

One of the parameters that is difficult to estimate is the murder rate $m$ of the gang members. Let us now turn our attention to Figures 9 and 10. We observe that for smaller values of $m$, the core gang population survives and reaches the coexistence equilibrium. However, we see that larger $m$ values will drive the core population to local extinction. The critical level of murder rate $m$ is between $2 * m$ and $2.5 * m$ and represents the difference between the existence and extinction of core gang members.

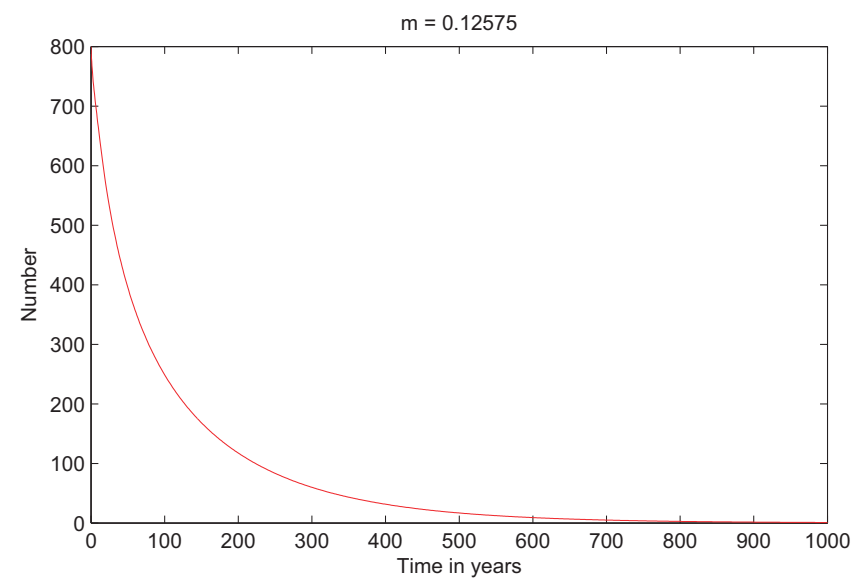

Figure 9. Graph showing the effect of $2.5 * m$ on $G$

There is a transcritical bifurcation at $\beta_{1}=0.256477$, any value lower than this will result in $G=0$. When

$$
\alpha+\mu(=0.12909)<\beta_{1}<0.256477, R_{0}>1,
$$

the system tends to the core-free equilibria (where $G, R=0$ and $n, s \neq 0$ ) and for values of $\beta_{1}<\alpha+\mu$, the system tends to the gang-free equilibrium (where $S, G, R=0$ and $n=1$ ).

Using the expression for $R_{0}$, a similar analysis may be done for $\alpha$. There is an lower bound $\alpha=0.354759$, and any value higher than this will result in $G=0$. When

$$
0.354759<\alpha<\beta_{1}-\mu(=0.70742), R_{0}>1,
$$

the system tends to the core-free equilibria (where $G, R=0$ and $N, S \neq 0$ ). However, for values of

$$
\alpha>\beta_{1}-\mu(=0.70742), \quad R_{0}<1
$$


the system tends to the gang-free equilibrium (where $S, G, R=0$ ). Branch points were also found at $\beta_{2}=$ 0.127845 and $\phi=0.265987$, meaning that for

$$
\beta_{2}<0.127845, \phi>0.265987,
$$

we obtain $G=0$, and the system tends to the core-free equilibrium.

\section{Discussion}

In developing this model, as far as possible, we tried to reflect the dynamics of joining a gang and desistance from gangs. The model considers risk factors for gang membership but does not consider causal factors for gang membership or gang activities such as violence or gun and drug sales. The contagiousness of the gang lifestyle (Katz \& Fox, 2010) plays a central role in allowing gangs to be treated as an infectious disease. In order to describe this contagiousness, the model contains two nonlinear standard incidence transmission terms. These terms represent recruitment rates into the gang. Indeed,to ensure continuity, gang members must actively recruit and retain its members (Sieberg, 2005). One of the more popular gang recruitment techniques has been described as "seduction or courting" with the promise of money, sex, and glamour (Harris, 2004). This suggests that an analogy may be made between recruitment of gang members and that of obtaining sexual partners. Standard incidence is generally used to describe the contact rate in sexually transmitted diseases (Mollison, 1995). Thus, standard incidence is chosen as the form for the contact rates.

The approach to reducing gangs involves prevention, intervention and suppression strategies (Spergel et al., 2006). Prevention programs target those at risk of gang involvement - the potential gang members $(N)$; intervention programs and strategies target fringe gang members $(S)$ so as to separate them from gangs; and law enforcement suppression strategies are aimed at the core gang members $(G)$ (Howellm, 2000). In this model, the prevention and intervention programs are related to $\alpha, \beta_{1}$ and $\beta_{2}$. Suppression strategies will be inherent in $\Phi, \rho$ and $f$.

This model has three possible equilibrium states, one which involved no gang members whatsoever, one which contained no core gang members and a coexistence equilibrium involving all the subpopulations in the model. In initiating antigang measures, the first state is highly desirable. However, this state occurs when the basic reproduction number $R_{0}$ is less than one - if the recruitment rate into the gang is less than the rate at which the members can be dissuaded from that lifestyle. The no-core members and the coexistence equilibrium occur if $R_{0}$ is more than one, with the no-core gang members clearly the preferable equilibrium of the two end states. Taking prevention, intervention and suppression strategies into consideration by changing all the parameters by $25 \%$ in combination and doubling the jail sentences led to the gang-free equilibrium.

One of the "quick fix" approaches to "solving" gang problems is increased suppression activities (Bleecker \& Town, 2008). However, this approach is essentially the treatment of the symptoms rather than the causes of youth gangs. Suppression in this model refers to the role of the criminal justice system in apprehending, convicting and rehabilitating core gang members. The Criminal justice system includes the police, the courts, and the jails. In the numerical simulations, increasing aspects of the criminals justice system like imprisonment rate (and hence deterrence), length of prison sentences $(\rho)$ and decreasing in recidivism rate do not have major impacts on the number of core gang members. This is because the are not related to the formation of the gang members. An increase in the imprisonment rate (and hence deterrence) of $25 \%$ with the length of prison sentences $(\rho)$ doubled and as well as a $25 \%$ decrease in recidivism rate leads to a decrease in core members of $35 \%$. This is a relatively small change considering the number of parameters changed. We consider this as related to improving the criminal justice system. If in addition to these measures, we decrease $\beta_{1}$ by $40 \%$, we reach the core-free equilibrium. If in addition to the changes in the criminal justice system, we decrease $\beta_{2}$ by $20 \%$, we reach the core-free equilibrium. This shows the importance of the $\beta_{2}$ parameter where relatively small changes in contact rates leading to the formation of a new core gang member leads to a big change in the core gang members.

In fact, reducing the contact rate $\beta_{2}$ between $S$ and $G$ has a bigger impact on the number of core gang members than a similar reduction in the contact rate $\beta_{1}$ between $N$ and $S$. Decreasing $\beta_{2}$ also resulted in an increase in fringe gang members, since fewer of them are becoming hard core members. This can be done by disrupting the recruitment process, though 'insulating' fringe gang members may prove difficult in communities where the gang lifestyle is deeply already entrenched since "joining a gang is like a rite of passage, especially for young men growing up in communities susceptible to this type of activity" (Sloane-Seale, 2010). The importance of this parameter has been recognised-in Trinidad and Tobago, the Anti-Gang Act 2011 makes it is illegal to recruit members for a gang with imprisonment for 20 years. 
The model does not consider youth gang exit programs-there is no flow out of $G$ into $S$ or $N$. This was because as far as we are able to determine, there are very few programs targeting youth who wish to leave a gang (Hastings et al., 2011). However, future work could include a category from $G$ into $N$. Law enforcement officials may be subject to the linear assumption of cause and effect-the expectation that an increase in policing effort will produce a corresponding improvement in crime. However when the bifurcation points are reached, any change in the parameters beyond that point will yield the same end results of states with no gang members. This means that devoting extra resources to say deterrence will work-up to a point, the bifurcation point.

There is a transcritical bifurcation at $\beta_{1}=0.256477$, any value lower than this will result in $G=0$. For $0.12909<$ $\beta_{1}<0.256477$, where $R_{0}>1$. The system tends to the core-free equilibria and for values of $\beta_{1}<0.12909$, the system tends to the gang-free equilibrium. From a practical point of view, in which the focus is to eliminate gangs, this means that there is a lower bound on $\beta_{1}$. This suggests that if the aim is to eliminate gangs completely, then one should reduce $\beta_{1}$ to values below $\alpha+\mu=0.12909$. Since $\alpha$ is included in the denominator in $R_{0}$, we also find a bound $\alpha=0.354759$, where any value higher than this will result in either the core-free or the gang-free equilibrium depending on the value of $R_{0}$. For $0.354759<\alpha<0.70742, R_{0}>1$ and the system tends to the core-free equilibria. For values of $\alpha>0.70742$, where $R_{0}<1$ the system tends to the gang-free equilibrium. Transcritical bifurcation points are also found at $\beta_{2}=0.127845$ and $\phi=0.265987$, where for $\beta_{2}<0.127845$ and $\phi>0.265987, G=0$ the system tends to the core-free equilibrium. Accordingly, by manipulating $\alpha$ and $\beta_{1}$, it is possible to totally eliminate gangs from the population. Is it possible for gangs to kill each other off? There is a bifurcation point at $m=0.1178=\frac{0.1178}{0.0503} \approx 2.34$ times the calculated murder rate, so that when this value is exceeded, $G=0$ or the core gang members effectively kill each other off.

Since controlled experiments cannot be performed on the system and the nature of the research is one in which the data is not easily available, one of the key challenges in this research lies in validating the model. In the spirit of Campbell and Ormerod (2001), we model for insights into key parameters and their causes and effects. We see that by changing the parameters by a relatively small amount, we are able to generate a wide range of values for the number of gang members. Parameters values and gang sizes vary globally. The results generated by changing parameters agree with key stylized facts on reduction strategies to treat gangs i.e. a combination of strategies is recommended. Though this agreement in no way validates the model, it at the very least makes the model credible. Building a crime model involves a multidisciplinary approach so as to bridge the gap between the natural and the social sciences. The "ideal type of the division of labor in quantitative social science would be one where the sociologist formulates a theory, the mathematician translates it into a mathematical model, and the statistician provides the tool for estimating the model"(Backman \& Edling, 2009).

\section{Conclusion}

To the best of our best knowledge, ours is the first attempt to model the complex, relatively recent social problem of gangs in Trinidad and Tobago. Though, criminal gangs make up a small proportion of the population, their effect on society is at odds with their size. Gang members are responsible for a disappropriately large number of violent crimes, and keep communities and in general the population in a state of fear.

Dr. Gary Slutkin, a Chicago epidemiologist (Austin, 2003) views gang violence as an infectious disease, and believes that the cure lies in changing behavior instead of increasing punitive measures. The model presented in this chapter treats the desire to join a criminal gang as an infectious disease motivated by peer pressure. The analysis shows the existence of three equilibrium states - two of which do not contain any gang members. Research on gangs (Wyrick \& Howell, 2004), Spergel et al. (2006) suggests that the most effective ways to reduce gangs is a combination of prevention, intervention and suppression strategies. The parameters in the model reflect aspects of these strategies and the greatest decrease in gang numbers involves changing all the parameters in combination. Bifurcation analyses performed showed the existence of bifurcation points where the behavior of the non-linear system changed to another state. Law enforcement officials and policy makers in developing anti-gang strategies and allocating funds need to be cognizant of the non-linear nature of the system where increasing crime fighting efforts may not lead to a corresponding decrease in gangs and where small changes in policies may make big differences in gang numbers and vice versa.

Trinidad and Tobago is a key transshipment point between the drug producers of South America and their markets in the North and between the gun markets of the USA and their markets in South America. Drugs, gangs and guns are inextricably linked (Trinidad and Tobago House of Representatives, Parlimentary session January 2008). Further research can include the effects of guns and drugs in the model by dividing the gang members into those 
involved in the drug trade-who should be more violent since they have easier access to weapons - and those who are not involved and therefore less violent.

\section{Appendix A}

$$
\begin{aligned}
& a_{1}: \alpha+3 \mu+\epsilon+\rho+H \beta_{1}+H \beta_{2}+K\left(\beta_{1}-\beta_{2}\right)-M \beta_{1}, \\
& a_{2}:-H \beta_{2}\left(q-K \beta_{2}+M \beta_{1}\right)-\left(H \beta_{1}+K \beta_{1}\right)\left(\alpha-M \beta_{1}\right)-\Phi \rho(-f+1)-\left(-\epsilon+K \beta_{2}\right)\left(\alpha+2 \mu+H \beta_{1}+H \beta_{2}+K \beta_{1}-M \beta_{1}\right) \\
& +\left(-\mu-H \beta_{1}-K \beta_{1}\right)\left(-\alpha-\mu-H \beta_{2}+M \beta_{1}\right)+(-\mu-\rho)\left(-\alpha-2 \mu-\epsilon-H \beta_{1}-H \beta_{2}-K \beta_{1}+K \beta_{2}+M \beta_{1}\right), \\
& a_{3}:-\Phi \rho\left(-\epsilon+K \beta_{2}\right)(-f+1)-H \beta_{2}\left(q-K \beta_{2}+M \beta_{1}\right)\left(\alpha+2 \mu+H \beta_{1}+H \beta_{2}+K \beta_{1}-M \beta_{1}\right) \\
& -H \beta_{2}\left(\left(H \beta_{1}+K \beta_{1}\right)\left(-q-M \beta_{1}\right)+\left(q-K \beta_{2}+M \beta_{1}\right)\left(-\alpha-\mu-H \beta_{2}+M \beta_{1}\right)\right) \\
& +\Phi \rho(-f+1)\left(-\alpha-2 \mu-\epsilon-H \beta_{1}-H \beta_{2}-K \beta_{1}+K \beta_{2}+M \beta_{1}\right) \\
& -\left(-\epsilon+K \beta_{2}\right)\left(-\left(H \beta_{1}+K \beta_{1}\right)\left(\alpha-M \beta_{1}\right)+\left(-\mu-H \beta_{1}-K \beta_{1}\right)\left(-\alpha-\mu-H \beta_{2}+M \beta_{1}\right)\right) \\
& -(\mu+\rho)\left(\begin{array}{c}
H \beta_{2}\left(q-K \beta_{2}+M \beta_{1}\right)+\left(H \beta_{1}+K \beta_{1}\right) \\
\left(\alpha-M \beta_{1}\right)+\left(-\epsilon+K \beta_{2}\right)\left(\begin{array}{c}
\alpha+2 \mu+H \beta_{1} \\
+H \beta_{2}+K \beta_{1}-M \beta_{1}
\end{array}\right) \\
-\left(-\mu-H \beta_{1}-K \beta_{1}\right)\left(-\alpha-\mu-H \beta_{2}+M \beta_{1}\right)
\end{array}\right) \\
& a_{4}:-\Phi\left(H \beta_{2}\left(f \rho\left(H \beta_{1}+K \beta_{1}\right)+\rho\left(q-K \beta_{2}+M \beta_{1}\right)(-f+1)\right)+\rho\left(-\epsilon+K \beta_{2}\right)^{2}(-f+1)\right) \\
& +\Phi \rho\left(-\epsilon+K \beta_{2}\right)(-f+1)\left(-\alpha-2 \mu-\epsilon-H \beta_{1}-H \beta_{2}-K \beta_{1}+K \beta_{2}+M \beta_{1}\right) \\
& +\Phi \rho(-f+1)\left(\begin{array}{l}
H \beta_{2}\left(q-K \beta_{2}+M \beta_{1}\right)+\left(H \beta_{1}+K \beta_{1}\right)\left(\alpha-M \beta_{1}\right)+\left(-\epsilon+K \beta_{2}\right)\left(\begin{array}{c}
\alpha+2 \mu+ \\
H \beta_{1}+H \beta_{2}+K \beta_{1}-M \beta_{1}
\end{array}\right) \\
-\left(-\mu-H \beta_{1}-K \beta_{1}\right)\left(-\alpha-\mu-H \beta_{2}+M \beta_{1}\right)
\end{array}\right) \\
& +(-\mu-\rho)\left(\begin{array}{c}
H \beta_{2}\left(q-K \beta_{2}+M \beta_{1}\right)\left(\alpha+2 \mu+H \beta_{1}+H \beta_{2}+K \beta_{1}-M \beta_{1}\right) \\
\left.+H \beta_{1}+K \beta_{1}\right)\left(-q-M \beta_{1}\right) \\
+\left(q-K \beta_{2}+M \beta_{1}\right)\left(-\alpha-\mu-H \beta_{2}+M \beta_{1}\right)
\end{array}\right)
\end{aligned}
$$

\section{Appendix B}

\section{Appendix B.1: Estimating T}

Gang membership is comprised of mainly young men. It has been estimated that males make up 95 per cent of gang members in Trinidad and Tobago (Hansard Parlimentary Debates, 2008), and that the average age of gang members ranges between fourteen (14) years and forty-four (44) years (Hansard Parlimentary Debates, 2008). Also, the average age of murder victims is 33 years (Maguire et al., 2008). Bearing this is mind and the classifications by age groups in the available statistical information, the population under consideration consists of male youth aged between 15-35 years living in areas where approximately $80 \%$ of gangs exist (Katz, 2009). Using census data from 2000 (Central Statistical Office, 2006), the number of male youth in area of interest $=101,870$.

The National Poverty Rate is the percent of the population of a country which earns less than that country's national poverty line-TT $\$ 665$ per month. In 2007, this was approximately 17.5\% (Ministry of Social Development, 2007). Thus, we use a base population from the bottom fifth (i.e. the poorest 20 per cent) of this male age group, $T=20,000$.

\section{Appendix B.2: Finding $S(0), R(0), G(0)$}

We decided to consider fringe gang members as dropouts from school and low achievers since research has shown that "youth with a low level of school commitment and low academic achievement have also been found to be significantly more likely to join a gang"considered easily quantifiable based on the limited data set available to us. Using data from (UNICEF, 2008), $S(0)=11092$.

The official statistics did not differentiate gang members in jail from other prisoners. However research by (Katz, 2009), estimated that $51.4 \%$ of gang members have been arrested, though there is no indication of whether they went to jail or not. Using this information and data of prisoners who commit serious crime from (Central Statistical Office, 2006), we use $R(0)=644$. 
Finally, extrapolating data from Hansard Parlimentary Debates (2008) and Katz (2009), we calculate that $G(0)=$ 800 .

\section{Appendix B.3: Calculating The Recidivism Rate $1-f$}

According to the Ministry of National Security (Ministry of National Security, 2008), the recidivism rate is about $56 \%$ for all crime. Let us assume that this refers to gang members as well. Since it is difficult leave a gang, this figure may in reality be greater than that. Therefore, we may safely take $f=0.44$.

\section{Appendix B.4: Calculating The Average Jail Sentence}

Gangs are most frequently involved in drug sales, robbery and firearms activity (Katz, 2009). All of these carry a minimum sentence of 5 years. Due to data limitations, we will not be considering inmates of detention facilities or people on probation. In a study of Recorded Murders and Persons Indicted for Murder in 1998-2002 (Hood \& Seemungal, 2006), it was observed that for gang crime, the death penalty is hardly enforced, as perpetrators are usually immune from the police system. In 2006, $89 \%$ of male jail sentences were 5 years and less, therefore we use $\rho=0.2$.

\section{Appendix B.5: Calculating The Imprisonment Rate}

This represents the proportion of gang members who go to jail per year. Though this appears simple enough, because of the lack of available data, it is relatively difficult to determine and we had to make a few assumptions to assist in its determination, which are

1. Gang members commit serious crimes- drug sales, robbery and firearms activity (Katz, 2009). According to the classifications of serious crime in the 2006 crime statistics published by Central Statistical Office of Trinidad and Tobago, the following reported crimes were considered: murder, woundings and shootings, kidnapping, robberies, firearm offences and narcotics offences.

2. Thus when calculating number of convictions, only convictions related to these are considered - murder, manslaughter, wounding, robbery, narcotics offences and other crimes.

3. Due to nature of serious crimes, all convicted youths go to jail since the crimes that they commit are considered indictable offences with a minimum jail sentence of five years (Central Statistical Office, 2006). Hence we take

$$
P(\text { jail sentence / convicted })=1 \text {. }
$$

4. There is negligible change in the ratio of serious crimes to convictions yearly.

5. Police crime statistics are based on cases that are reported to the police by the public and thus there might be significant under-reporting of offences.

6. The judicial process is the same in both Trinidad and Tobago.

7. Gangs were responsible for 53\% of all murder victims in 2003-2007 (Magazine, 2008). We are assuming that this figure also holds for the serious crimes under consideration.

The information used in this section was obtained from the Annual Statistical Digest Of Trinidad and Tobago for 2006 (Central Statistical Office, 2006). The Rate at which gang members go to jail is

$$
P(\text { convicted }) * P(\text { jailsentence/convicted }) * n
$$

where $n$ is the number of reported serious crimes attributed to a gang member per gang member. From the data provided, the rate at which gang members go to jail is found to be $\Phi=0.115$.

\section{Appendix B.6: Calculating $\alpha$}

It is assumed that the interference parameter $\alpha$ is related to $\Phi$ the imprisonment rate, since swift and effective justice may deter youths from a life of crime (Harries, 2003). In addition to deterrence, intervention programs may be used to reduce the criminal activities of gangs by offering alternative opportunities for youth and may include rehabilitation measures (Howell, 2000). Hence we $\alpha \geqslant \Phi$, and we get $\alpha=1.1 * \Phi=1.1 * 0.11501=0.12651$. 


\section{Appendix B.7: Calculating $\beta_{1}$ and $\beta_{2}$}

The term "contact" means an interaction-either direct or indirect-between individuals sufficient for disease transmission. This depends on the mode of transmission. Contact rates are difficult to estimate, because of the inherent difficulty in describing what constitutes a contact and what does not. Contact in this model is different from that in other epidemiological models. Here contact can mean both a direct contact and an indirect one. Direct in the sense of active recruitment and indirect in the sense that individuals may want to emulate gang members because of their flashy lifestyle.

The model has two types of contact $\beta_{1}$-candidates rate-from $N$ to $S$ and $\beta_{2}$-conversion/recruitment rate- from $S$ to $G$. There is an assumption is that it is harder to convince a potential gang member to take the final step and join the gang than it is to persuade someone to consider joining a gang (recruit) $\beta_{1} \geqslant \beta_{2} \geqslant 0$. Recall $\beta_{t}(i=1,2)$ is the number of contacts needed to transmit infection (to a susceptible) in unit time per infective.

Using information from (Katz, 2009), 25.8\% of gangs formed before 2000. 74.2\% of gangs formed between 20002006. Assume that in $2000 \frac{74.2 \%}{6}$ of gangs were influenced to form by the $25.8 \%$. If $N$ is the total number of gangs up to 2006, then in the year 2000, $\frac{74.2}{6 * 100} N$ gangs were influenced by $\frac{25.8}{100} N$ gangs, This means that 1 gang was influenced by $\frac{25.8 * 6}{74.2}$ gangs. We therefore calculate the number of contacts that result in the formation of a new gang to be $\frac{25.8 * 6}{74.2}=2.0862$. However, this represents the contact rate with the entire gang, and we need to know the contact rate per member. Taking the average number of members per gang in Trinidad to be 10 , the number of contacts per member per year is

$$
\beta_{2}=\frac{2.0862}{10}=0.20862=0.21
$$

The number of gang members for each successive year will be influenced by an increasing number of gangs since we assume that the number of gangs increases by $\frac{74.2 \%}{6}$. Hence we use this value as a minimum one.

Convincing someone to consider joining a gang $\beta_{1}$, may be done both directly and indirectly - societal approval of gang members plays a part here, since some of the gang members are seen as modern day "Robin Hoods". Their supporters include family members who accept cash from their relatives without wondering about their source, the young women who are attracted to the gang members by the trickle-down wealth and prestige, and the children of the communities who observe the fast money and respect accorded to these gangsters (Katz, 2009).

Societal pressure is thought to operate in two ways:

1. The greater the proportion of gang members in any given population, the more likely it is that any individual will convert. Conversely, the greater the proportion of the population who are not interested in being part of a gang, the greater the pressure on those who are criminals to become law-abiding.

2. Gang's also provide a "role in informal social control within the community" since gang leaders police their territory and take care of the people in the community (Katz, 2009).

Let us assume that to convince a non-susceptible member to consider joining a gang would at least as much contacts as to convince a potential recruit to join the gang. Also since we are assuming that $\beta_{1} \geqslant \beta_{2}$, we must consider the additional influence of societal pressure. Now

$$
\beta_{1}=\beta_{2}+a=0.21+a
$$

where $0 \leq a \leq 1$, where $a=0$ represents total societal disapproval, and $a=1$ total societal approval. We may therefore assume that $\beta_{1} \geqslant \beta_{2} \geqslant 0$. It should be noted that this factor is difficult to measure in practice. If we assume that the community is indifferent to the gang and turning a blind eye to their activities, then $a=0.5$ gives $\beta_{1}=0.71$, and we may conclude that $0.21 \leq \beta_{1} \leq 1.21$.

Appendix B.8: Calculating A, $\mu, m, q$

$A$ : Number of incoming youth to the population

$A$ is the number of youth turning 14 in the geographical area of interest. Using (Central Statistical Office, 2006) to obtain birth rates, this is estimated at 750 . 
According to the population statistics, up to 1999, the number of murders per year was fairly constant at about 100. Let us assume that these murders were not gang related. Hence we will use the averages from 1995-1999 for males 15-34 years. This gives $\mu=2.585 \times 10^{-3}$.

Up to 1999 , the number of murders was fairly constant at about 100. St. Bernard (St. Bernard, 2009) noted that gang-related and drug-related activities homicides were generally associated with the use of a firearm. The percentage of homicides due to firearms has been steadily increasing since 2000. From 2004 - 2008, approximately $75 \%$ of all homicides involved the use of a firearm compared with 58\% between 2000-2003. Let us assume that for each member of the public killed by a gun, a gang member is killed. Since the number of murders in the year 2007 was 391, and the number of deaths due to firearms was 303, we may assume $25 \%$ of those killed were gang members, which gives the number of gang members murdered to be $0.25 * 303=75$. 75 . It follows that

$$
m=\frac{\text { Murdered gang members }}{\text { Number members }}=\frac{75.5}{1500}=0.0503
$$

We note that $\frac{0.0503}{0.00258}=20$ times the natural death rate.

Though this may appear to be high, research in the United States found that when considered nationally, a youth gang member's risk of being killed is 60 times greater than that of the general population (Morales, 1992). In St. Louis, the gang member homicide rate is 1,000 times higher than the U.S. homicide rate (Decker \& Winkle, 1996). They found that the number of firearm related homicides are increasing. Hence the value calculated for the murder rate may in reality be a lower bound.

Many street gang members appear to be predestined for gang membership as they may have family members siblings, parents, uncles or cousins or friends in a gang who they view as role models. The average male birth rate per 1000 persons is 7.249. Let us apply this birth rate to gang members and also assume that each gang member has one family member/friend who is turning fourteen whom he can influence to join the gang. This may be considered as his "adopted child". Hence

$$
q=(7.249 * 2) / 1000=1.4498 \times 10^{-2} .
$$

\section{References}

Austin, E. (2003). Treating violence as a contagious disease.

Backman, O., \& Edling, C. (1999). Mathematics matters: On the absence of mathematical models in quantitative sociology. Acta Sociologica, 42, 69-78. http://dx.doi.org/10.1177/000169939904200105

Bernard, G. S. (2009). Firearms and reported homicide in Trinidad and Tobago: A socio-demographic perspective (unpublished).

Bingenheimer, J. B., Brennan, R. T., \& Earls, F. J. (2005). Firearm violence exposure and serious violent behavior. Science, 308, 1323-1326. http://dx.doi.org/10.1126/science.1110096

Bleecker, W., \& Town, R. (2008). Report to the legislature findings and recommendations of the gangs in schools task force. Gangs in Schools Task Force.

Brown, C. (1995). Serpents in the sand: Essays on the nonlinear nature of politics and human destiny. University of Michigan Press.

Campbell, M., \& Ormerod, P. (1997). Social interaction and the dynamics of crime.

Castiglione, F. (2006). Agent based modeling. Scholarpedia, 1(9), 1562. http://dx.doi.org/10.4249/scholarpedia.1562

Castillo-Chavez, C., Blower, S., van den Driessche, P., Kirschner, D., \& Yakubu, A.-A. (2002). Mathematical approaches for emerging and reemerging infectious diseases. An introduction to models, methods, and theory, 1. New York: Springer-Verlag.

Central Statistical Office. (2006). Annual statistical digest. Central Statistical Office of Trinidad and Tobago.

Decker, S., \& Winkle, B. (1996). Life in the gang: Family, friends, and violence. Cambridge criminology series. Cambridge University Press. http://dx.doi.org/10.1017/CBO9781139174732

Dhooge, A., Govaerts, W., \& Kuznetsov, Y. A. (2003). Matcont: A matlab package for numerical bifurcation analysis of odes. ACM Trans. Math. Softw., 29(2), 141-164. http://dx.doi.org/10.1145/779359.779362 
Gordon, M. (2010). A random walk in the literature on criminality: A partial and critical view on some statistical analysis and modeling approaches. European Journal of Applied Mathematics, 21, 283-306. http://dx.doi.org/10.1017/S0956792510000069

Government of Trinidad and Tobago. (2008). Government considering legislation to prohibit formation of gangs. Technical Report.

Hagedorn, J. (2008). A world of gangs: Armed young men and gangsta culture. U of Minnesota Press.

Hansard Parlimentary Debates. (2008). Trinidad and Tobago House of Representatives official report. 2nd Sitting1st Session-9th Parliament.

Hansard Parlimentary Debates. (2008). Trinidad and Tobago House of Representatives official report. 4th Sitting1st Session-9th Parliament.

Harries, R. (2003). Modeling and predicting recorded property crime trends in England and Wales, a retrospective. International Journal of Forecasting, 19, 557-566. http://dx.doi.org/10.1016/S0169-2070(03)00090-6

Harris, D. (2004). Gangland. Holy Fire Publishing.

Hastings, R., Dunbar, L., \& Bania, M. (2011). Leaving criminal gangs: Exit strategies and programs. Technical Report, Institue for the Prevention of Crime.

Hood, R., \& Seemungal, F. (2006). A rare and arbitrary fate: Conviction for murder the mandatory death penalty and the reality of homicide in Trinidad and Tobago. Technical Report, Centre for Criminology, University of Oxford.

Howell, J. C. (2000). Youth gang programs and strategies. Technical Report, Office of Juvenile Justice and Delinquency Prevention.

Howell, J. C. (2010). Gang prevention: An overview of research and programs. Juvenile Justice Bulletin, 1-22.

Katz, C. M. (2009). Diagnosing gang problems in the Caribbean. CARICOM Conference on Violence Prevention.

Katz, C. M., \& Choate, D. (2009). Diagnosing Trinidad and Tobago's Gang Problem. Annual Meeting of the American Society of Criminology, Los Angeles, California.

Katz, C. M., \& Fox, A. M. (2010). Risk and protective factors associated with gang-involved youth in Trinidad and Tobago. Pan American Journal of Public Health, 27(3), 187-202. http://dx.doi.org/10.1590/S1020-49892010000300006

Klein, M. W. (1995). The American street gang: Its nature, prevalence, and control. Studies in crime and public policy. Oxford University Press, USA.

Maetens, F., \& Anstey, C. (2007). Trends, costs, and policy options in the Caribbean. Joint report of the United Nations Office on Drugs and Crime, and the Latin America and Caribbean Region of the World Bank. Technical Report 37820.

Magazine, E. (2008). A Caribbean crime wave.

Maguire, E. R., Wllis, J., Snipe, J., \& Gantley, M. (2008). Spatial concentrations of violence in Trinidad and Tobago. Caribbean Journal of Criminology and Public Safety, 13, 48-92.

Ministry of National Security. (2008). Crime solving capability. Technical Report, Trinidad and Tobago Police Service.

Ministry of Social Development of Trinidad and Tobago. (2007). Puente in the Caribbean overview-Trinidad and Tobago.

Mollison, D. (1995). Epidemic models: Their structure and relation to data. Newton Institute. Cambridge University Press.

Moore, J. (1978). Homeboys: Gangs, drugs, and prison in the barrios of Los Angeles. Temple University Press.

Morales, A. (1992). A clinical model for the prevention of gang violence and homicide. In R. Cervantes (Ed.), Substance Abuse and Gang Violence. Sage Publications.

Nawojczyk, S. (1997). Street gang dynamics. 
Ormerod, P., Mounfield, C., \& Smith, L. (2001). Non-linear modelling of burglary and violent crime in the UK. In C. Lewis (Ed.), Modelling Crime and Offending: recent developments in England and Wales. Vol 80. Research, Development and Statistics Directorate, Home Office of C. Lewis, London, UK.

Sieberg, K. K. (2005). Criminal Dilemmas: Studies in economic theory, 12. Springer Berlin, Heidelberg.

Sloane-Seale, G. (2010). A look at school and gang violence.

Spergel, I. A., Wa, K. M., \& Sosa, R. V. (2006). The comprehensive, community-wide, gang program model: Success and failure. AltaMira Press/Rowman \& Littlefield, Lanham, MD.

UNICEF (2008). Education statistics: Trinidad and Tobago. Technical report, UNICEF.

Wyrick, P. A., \& Howell, J. C. (2004). Strategic risk-based response to youth gangs. Juvenile Justice, 10(1), 20 -29. 